\title{
Feasibility of Speech Telerehabilitation for a Patient with Parkinson's Disease in a Low-Resource Country during the Pandemic: A Case Report
}

\author{
Francis Exequiel M. Laxamana, MD, ${ }^{*}$ Carl Froilan D. Leochico, PTRP, MD, ${ }^{1,2 *}$ Adrian I. Espiritu, MD, 3,4 \\ Gabrielle lonne T. Sy, ${ }^{2}$ Reynaldo R. Rey-Matias, MD, MSHMS ${ }^{1,2}$ and Roland Dominic G. Jamora, MD ${ }^{3,5}$ \\ ${ }^{1}$ Department of Physical Medicine and Rehabilitation, St. Luke's Medical Center - Quezon City, Philippines \\ ${ }^{2}$ Department of Rehabilitation Medicine, Philippine General Hospital, College of Medicine, University of the Philippines Manila, Manila, Philippines \\ ${ }^{3}$ Division of Adult Neurology, Department of Neurosciences, Philippine General Hospital, College of Medicine, University of the Philippines Manila, Manila, Philippines \\ ${ }^{4}$ Department of Clinical Epidemiology, College of Medicine, University of the Philippines Manila, Manila, Philippines \\ Institute for Neurosciences, St. Luke's Medical Center, Quezon City and Global City, Philippines
}

*Joint first authors

\begin{abstract}
Parkinson's disease (PD) is a chronic, neurodegenerative condition resulting in various motor impairments, including speech disorders. However, at the height of the coronavirus disease 2019 pandemic, a patient with PD could not access traditional in-person neurorehabilitation care. This case report highlights the feasibility of telerehabilitation to deliver speech therapy over a distance using available resources in a developing country.
\end{abstract}

We describe a Filipino elderly woman, public speaker, and marriage counselor, seeking teleconsultation for her voice problems (slow and soft) attributed to PD. At that time, most center-based outpatient rehabilitation centers in Manila were closed due to the pandemic, and the patient preferred to stay at home for safety reasons. Hence, she was evaluated and managed remotely by an interdisciplinary team (neurologist, physiatrist, speech-language pathologist) through video calls. Since the ideal rehabilitation set up (in-person evaluation and therapy; use of Lee Silverman Voice Therapy) could not be done, the clinicians had to find practical alternatives, such as remotely administering subjective perceptual voice assessments, objective speech analysis using the Praat ${ }^{\mathrm{TM}}$ computer application, and speech teletherapy through synchronous (videocalls, phone calls) and asynchronous (e-mails, text messages, prerecorded exercise videos) techniques.

Notable speech improvements were observed by the clinicians, patient, and patient's frequent communicative partners after at least four teletherapy sessions. However, the carry-over of the improvements was affected by the patient's lack of compliance with the prescribed home exercise program.

Telerehabilitation using synchronous and asynchronous techniques for speech disorders due to PD was found feasible, beneficial, safe, and practical amid social distancing and low resources in a developing country.

Keywords: Speech therapy, telerehabilitation, teletherapy, parkinson's disease, voice disorders, coronavirus disease 2019, acoustic measurement, Lee Silverman Voice Therapy

\section{INTRODUCTION}

Corresponding author: Carl Froilan D. Leochico, PTRP, MD Department of Physical Medicine and Rehabilitation St. Luke's Medical Center - Quezon City, Philippines

\section{Department of Rehabilitation Medicine} Philippine General Hospital College of Medicine University of the Philippines Manila Taft Avenue, Ermita, Manila 1000, Philippines Email: cfdleochico@stlukes.com.ph
Parkinson's disease (PD) is a complex and chronic neurodegenerative disease caused by severe loss of nigrostriatal dopaminergic neurons. ${ }^{1,2}$ Aside from limb manifestations (e.g., tremors, bradykinesia), voice disorders occur in approximately $90 \%$ of patients with $\mathrm{PD}$ in the form of hypokinetic dysarthria, characterized by alterations in articulation, phonation, prosody, speech fluency, and faciokinesis. ${ }^{3}$ Thus, severe disability may be seen among patients with $\mathrm{PD}$ as voice disorders may worsen the quality of life. ${ }^{4}$ 
Speech defects as part of PD may contribute to the development of dementia, depression, and apathy. ${ }^{5} \mathrm{~A}$ comprehensive rehabilitation approach, consisting not just of physical therapy and occupational therapy, but also of speech therapy, is imperative for patients with PD. The goals for speech therapy may include, but are not limited to: (a) defining the patient's speech concerns, (b) evaluating voice quality, (c) recommending appropriate speech interventions, and (d) educating patients on energy conservation and breathing techniques. ${ }^{5}$

The evaluation and management of speech disorders in PD have been challenging due to inconsistent evidence in the literature. ${ }^{6-8}$ To provide a more objective and consistent evaluation of acoustic parameters, the Praat ${ }^{\mathrm{TM}}$ computer software application (Boersma, 2011) has been cited in the literature. ${ }^{9}$ The accuracy and utility of Praat ${ }^{\mathrm{TM}}$ in quantitatively characterizing the speech of patients with PD have been reported..$^{10,11}$ Furthermore, to provide more holistic and effective management for these patients, multimodal treatment is advocated, consisting of but not limited to the following: neuropharmacological therapies (e.g., levodopa, clonazepam); neurosurgical procedures (e.g., deep brain stimulation); and behavioral in-person or virtual speech therapy (e.g., Lee Silverman Voice Therapy or LSVT). ${ }^{6}$ The LSVT has been used and well-researched for almost 20 years and has become the standard of care, employing intensive respiratory-phonatory exercises aimed at improving vocal quality, volume, intonation, and articulation among others. ${ }^{6,12-14}$ Unfortunately in the Philippines, it has not been commonly employed even before the coronavirus disease 2019 (COVID-19) pandemic because of several reasons, such as potential costs, lack of awareness of patients and clinicians, and scarcity of LSVT-certified speech therapists (i.e., only 4 locally based on the LSVT website). ${ }^{15,16}$

With the pandemic that has further limited the in-person access to rehabilitation services, patients and clinicians have turned to telerehabilitation (i.e., use of telecommunication technologies for consultation and therapy) amid the limited resources in a developing country, such as the Philippines. ${ }^{17,18}$ However, performing speech evaluation and management on a patient with PD, in particular, has neither been widely practiced nor documented. This case report, therefore, aimed to document the feasibility of telerehabilitation as alternative speech therapy for PD in a low-resource country during the COVID-19 pandemic. In addition, we share our clinical experience with the use of $\operatorname{Praat}^{\mathrm{TM}}$ as a measurement tool to evaluate the patient's speech objectively and virtually over a distance.

\section{CASE PRESENTATION, INTERVENTION, AND OUTCOMES}

A 61-year-old Filipino woman, working as a public speaker and marriage counselor, without comorbid conditions, was diagnosed with PD in 2018. She initially presented with resting tremors of the right foot, progressing to the arm with bradykinesia and illegible handwriting. She continued to be ambulatory with a single-tip cane and has had modified independence in activities of daily living (i.e., with adaptations in tasks and environment as safety precautions). She underwent regular physical therapy for maintenance of her functional mobility, balance, and endurance before the pandemic, and continued with home exercises during the pandemic. She has regularly followed up with her neurologist for several years and has been maintained on levodopa. Her latest chief complaint during the lockdown was slow and soft speech, for which she was referred to a physiatrist for telerehabilitation. Electronic informed consent was secured from the patient for teleconsultation and teletherapy sessions.

On initial teleconsultation with the physiatrist through video call, the patient was observed to be alert, able to follow commands, with unremarkable systemic tele-evaluation. She was ambulatory with functional strength on all limbs. Executive functions were intact. There was no bradyphrenia (abnormally slow mental processing), but bradykinesia (abnormally slow movement) and bradylalia (abnormally slow speech) were noted. Her voice had low pitch and amplitude. There were several occasions when family members and other frequent communicative partners had to ask her to repeat what she said for clarification. The patient admitted to having limited self-awareness of the quality and softness of her voice. Before the diagnosis of $\mathrm{PD}$, she claimed to have had good voice projection at work. After two years of having PD, however, she began experiencing difficulty with maintaining appropriate voice amplitude towards the end of the day attributed to fatigue. For instance, she had to schedule all her work-related speaking engagements in the morning as she and her peers noticed regression of her voice quality even by mid-day. She felt straining of her voice, characterized as hoarseness, throat discomfort, and effortful sound production. The patient was advised to continue her intake of levodopa and undergo speech teletherapy. She was referred to a speech-language pathologist privately practicing teletherapy. A perceptual assessment was initially used for subjective tele-evaluation. There was difficulty with the production of high-amplitude sounds and pitch variation during phonation. For objective tele-assessment, the Praat $^{\mathrm{TM}}$ application version 6.1.16 was used to quantify vocal abnormalities. Using the application, the patient was found to have a maximum phonation time (MPT) below normal (i.e., usually 5 seconds), and GRBAS (i.e., Grade, Roughness, Breathiness, Asthenia, and Strain) scale ratings of 1, 0, 0, 1, and 0 , respectively (scores for each GRBAS item range from 0 to 3 in increasing severity). ${ }^{19}$ Figure 1 shows a screenshot of the Praat ${ }^{\mathrm{TM}}$ interface, depicting the patient's speech graphical and numerical recording at baseline. Numerical results obtained from Praat ${ }^{\mathrm{TM}}$ before speech teletherapy are presented in Table 1.

The patient's pitch range improved as evidenced by the lower minimum and higher maximum values of pitch 


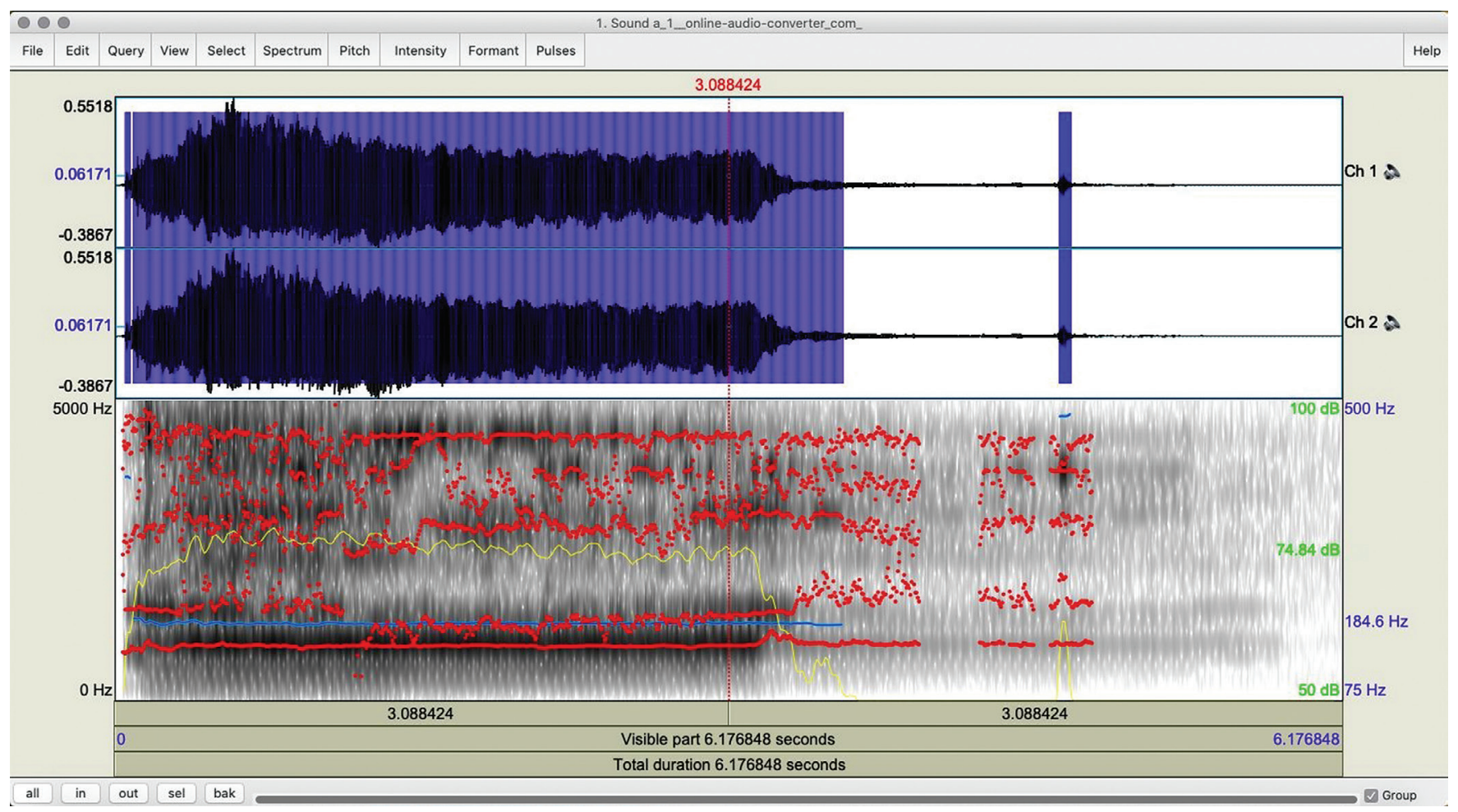

Figure 1. Screenshot of the interface of Praat ${ }^{\mathrm{TM}}$, showing the patient's speech record. The top panel with blue and black shades shows the patient's sound waveform. The bottom panel shows the sound's corresponding spectral energy (spectrogram) over time: red lines representing formants (overtones and timbre in Hertz, $\mathrm{Hz}$ ); blue line representing pitch (frequency, $\mathrm{Hz}$ ); yellow line representing intensity (volume, decibels, or $\mathrm{dB}$ ). The bottom-most bar when clicked plays the entire duration of the sound, while the middle bar plays only the visible portion of the sound, and the top bar plays a specific part in the visible sound depending on a chosen particular time point or cursor location (red vertical line corresponding to the topmost number in red). ${ }^{20}$

Table 1. Objective speech tele-assessment using Praat $^{\mathrm{TM}}$ before and after 8 teletherapy sessions

\begin{tabular}{lccccc}
\multicolumn{1}{c}{ Parameter (Unit) } & $\begin{array}{c}\text { Average/ } \\
\text { Normal Values }\end{array}$ & Pre-Treatment & Interpretation & Post-Treatment & Interpretation \\
\hline Mean Pitch (Hz) & 225 & 183.795 & $\begin{array}{c}\text { May vary per individual } \\
\text { (i.e., habitual pitch) }\end{array}$ & $\begin{array}{c}178.383 \\
\text { May vary per individual } \\
\text { (i.e., habitual pitch) }\end{array}$ \\
\hline Minimum Pitch (Hz) & 155 & 180.709 & Above average & $\begin{array}{r}78.393 \\
\text { Improved from baseline; } \\
\text { pitch range improved }\end{array}$ \\
\hline Maximum Pitch (Hz) & 334 & 191.318 & Below average & $\begin{array}{r}468.998 \\
\text { Improved from baseline; } \\
\text { pitch range improved }\end{array}$ \\
\hline Local Jitter (\%) & $\leq 1.040$ & 0.307 & Within normal limits & 0.873 & Within normal limits \\
\hline Local Shimmer (\%) & $\leq 3.810$ & 9.523 & Above average & 10.328 & Above average \\
\hline Mean Harmonics-to-Noise Ratio & $>20$ & 14.852 & Below average & 12.499 & Below average \\
\hline Mean Noise-to-Harmonics Ratio & $>1$ & 0.046 & Below average & 0.113 & Below average \\
\hline
\end{tabular}

post-treatment (Table 1). Clinically, the patient's voice was less monotonous with relatively better intonation, stress placement, and rhythm on follow-up. However, the patient's shimmer, also known as amplitude perturbation (i.e., the difference in amplitude from cycle to cycle), remained high or above average, as often seen in pathological voices. ${ }^{20}$ In addition, her harmonics-to-noise and noise-to-harmonics ratios (i.e., the amount of periodic noise compared to the amount of irregular, aperiodic noise, representing turbulent airflow in the vocal tract) remained below average, as observed in patients with persistent hoarseness. ${ }^{20}$ Nonetheless, her jitter (pitch perturbation) was within normal limits, as observed in either healthy or some pathological voices. ${ }^{20}$

The patient's pertinent speech findings on teleconsultations and corresponding teletherapy interventions and outcomes are summarized in Table 2. The speech 
Table 2. Patient's speech findings and rehabilitation course over 8 weeks of teletherapy

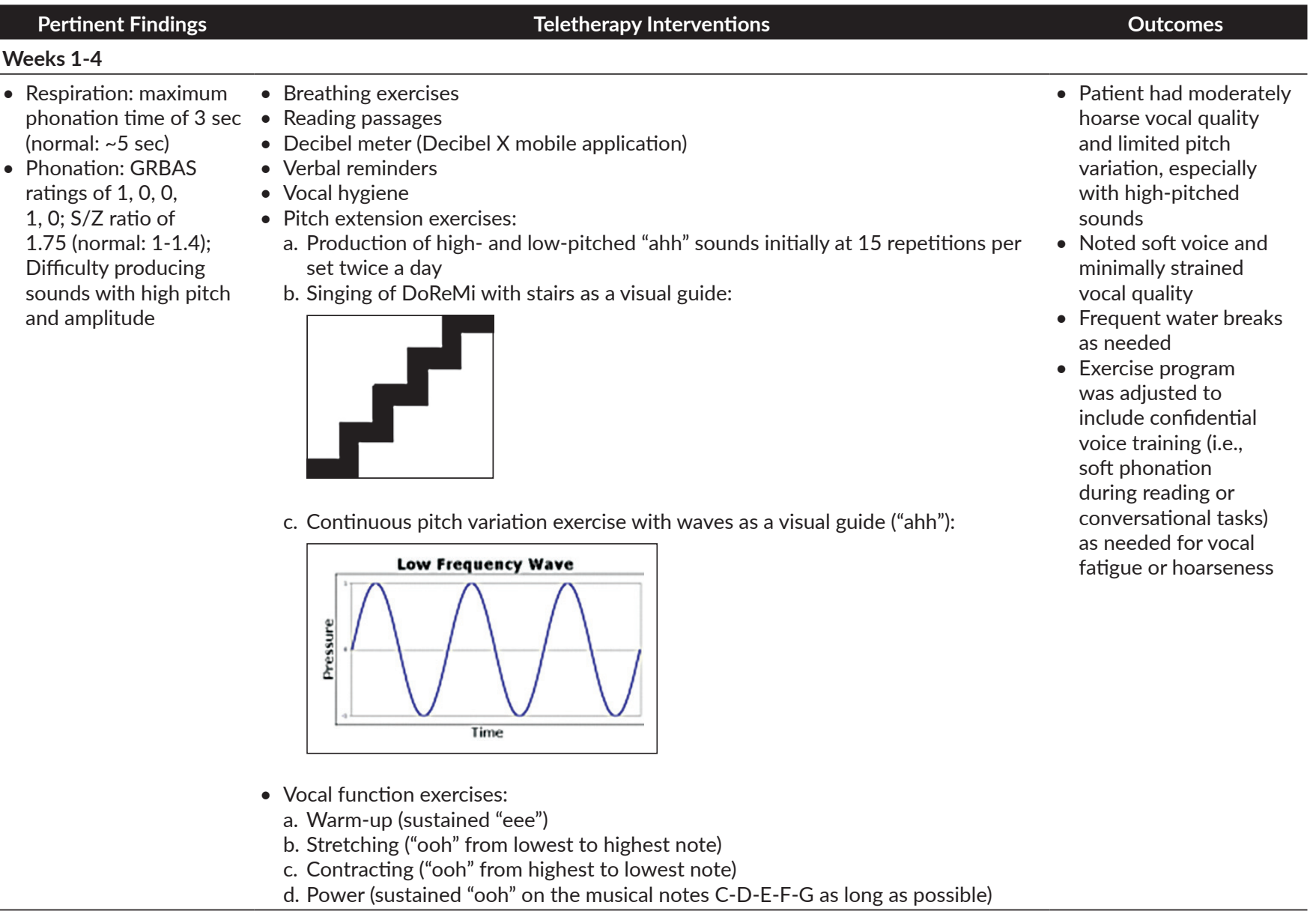

telerehabilitation program focused on vocal function and pitch extension exercises, principles of choral singing therapy, and principles of phonation resistance training exercises (PhoRTE). ${ }^{21}$

Telerehabilitation using low-cost, home-available electronic devices, and a freely downloadable video conferencing platform were used in two distinct ways: (a) teleconsultations with the physiatrist for initial and followup evaluations, and (b) teletherapy sessions with the speechlanguage pathologist. The link to the online meeting was sent by the clinician to the patient 20-30 minutes before the start of each session. The patient used her laptop and earphones with a microphone, while the clinicians used their laptops and, occasionally, mobile devices. The video camera and microphone were turned on throughout each session to allow the clinicians to synchronously evaluate the patient's performance of the tasks. Visual guides, such as photos and videos, were used as needed through the screen-sharing feature of the videoconferencing platform. The teleconsultations lasted for an average of 30 minutes done twice a month for two months, while the supervised teletherapy sessions lasted for an average of 1 hour once to twice a week for 8 weeks. Instead of the 2-3 times a week frequency of teletherapy sessions as recommended by the physiatrist, the patient eventually shifted from having sessions initially twice a week to just once a week, supplemented by asynchronous monitoring of home exercise program with pre-recorded exercise videos, due to costs.

After the first teletherapy session, specific home exercises for increasing voice volume and improving pitch variations were given. During follow-up, the patient was noted to have a relatively reduced voice volume. Aside from reiterating the need for compliance with the home exercises and engagement of a family member in ensuring that the exercises were done regularly, practical tips on voice hygiene were recommended, such as frequent water intake and rest periods in between long talks.

The patient had eight teletherapy sessions. Notable improvements were observed by the clinicians, patient, and patient's frequent communicative partners during the second week of teletherapy sessions. At the end of the $8^{\text {th }}$ week, the patient was re-evaluated and the following were recommended: (a) continued one-on-one remotely supervised speech and language intervention at least once a week, focusing on monitoring and maintaining communication skills, such as voice and phonation; (b) counseling on the 
Table 2. Patient's speech findings and rehabilitation course over 8 weeks of teletherapy (continued)

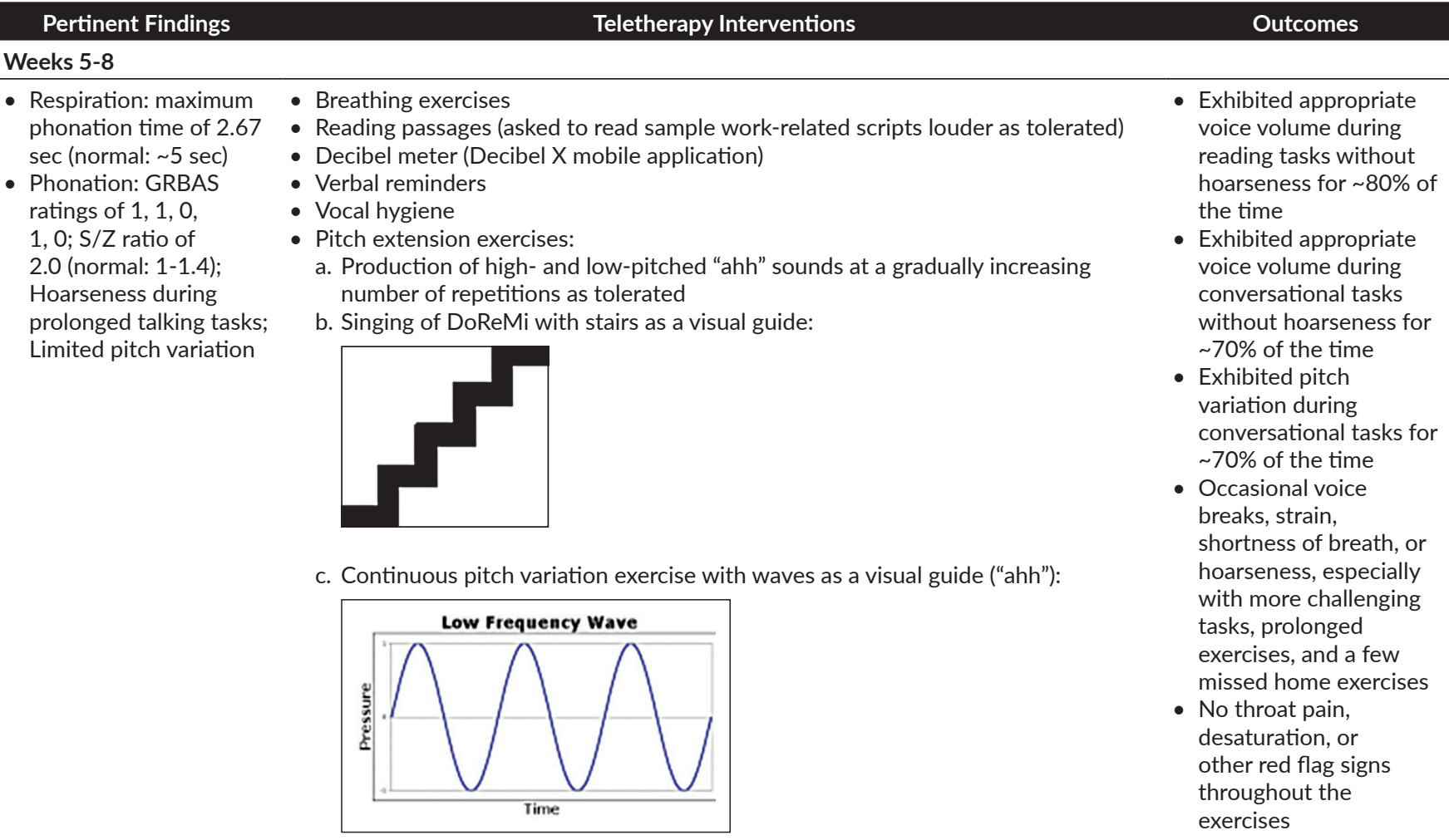

- Vocal function exercises:

a. Warm-up (sustained "eee")

b. Stretching ("ooh" from lowest to highest note)

c. Contracting ("ooh" from highest to lowest note)

d. Power (sustained "ooh" on the musical notes C-D-E-F-G as long as possible)

Sec: seconds. GRBAS (grade, roughness, breathiness, asthenia, strain) ratings: scored from 0-3 per parameter; directly proportional to voice severity. ${ }^{19}$ $S / Z$ ratio: how long one can sustain the voiceless " $s$ " and voiced " $z$ " sounds; higher values may indicate laryngeal pathology. ${ }^{22}$

results of the speech and language re-assessment using Praat $^{\mathrm{TM}}$ and the importance of appropriate and daily implementation of carry-over or home activities; and (c) continued regular medical consultations.

\section{DISCUSSION}

Speech telerehabilitation was found feasible for our patient with $\mathrm{PD}$, enabling us to provide prompt consultation and therapy services despite the physical distance due to COVID-19. We were able to administer speech evaluations through synchronous telerehabilitation both subjectively using perceptual assessments and objectively using Praat ${ }^{\mathrm{TM}}$. Our patient was able to benefit from synchronous and asynchronous teletherapy sessions, which resulted in improvements in pitch and vocal hygiene awareness. Irregular compliance to the home exercise program, however, could have contributed to the persistently soft voice and hoarseness, as reflected by high shimmer and low harmonicsto-noise and noise-to-harmonics ratios. Continued telerehabilitation was recommended.
In telerehabilitation, which is the innovative and remote delivery of usual Rehabilitation Medicine services, teleconsultations and teletherapy go hand-in-hand. ${ }^{23}$ Currently, there are only 259 fellows of the Philippine Academy of Rehabilitation Medicine and 673 members of the Philippine Association of Speech Pathologists. ${ }^{17}$ With the shortage of rehabilitation workforce and facilities that are mostly located in the urban areas in the Philippines, telerehabilitation can be leveraged to improve access to specialized services to patients, especially in remote areas. ${ }^{18}$ During the pandemic, even patients in urban areas, just like our patient, were unable to access rehabilitation services because of stringent quarantine and social distancing measures.

To our knowledge, there has been one published report on speech teletherapy for PD from a developing country in Asia. ${ }^{24}$ However, in the Philippines, there is no published report on speech teletherapy. LSVT via telerehabilitation had been used and found to be effective in treating speech disorders in PD. ${ }^{19}$ Although the LSVT remains to be standard of care in treating the speech disorders of $\mathrm{PD}$, there 
have been barriers to its implementation in the Philippines. Among these barriers are the lack of LSVT-certified speech therapists in the country, potential additional costs for each therapy session (i.e., anecdotally more expensive than usual speech therapy by approximately PHP 1,000.00, varying from one private practitioner or institution to another), expensive international certification training (i.e., at least approximately PHP 29,000.00), ${ }^{16}$ and lack of local training opportunities for clinicians. For our patient, the actual fee accrued for speech teletherapy per session was PHP 1,300.00.

Since teletherapy services were not available in the affiliated private hospitals of the physiatrist, the patient had to be referred to a freelance speech-language therapist offering teletherapy. Currently, there is no available directory of local physiatrists and allied rehabilitation professionals (e.g., physical/ occupational/ speech therapists) who conduct telerehabilitation in their practice. A directory could be a useful reference for referring doctors and patients in need of teletherapy. At the time of writing, it seems there are more local government tertiary hospitals and stand-alone therapy centers that have been offering telerehabilitation services compared to private tertiary hospitals. Most of the private hospitals in Metro Manila, Philippines have not yet adopted this kind of service possibly due to the lack of knowledge and acceptance of telerehabilitation and the lack of readiness and technical know-how to interact in a secure telemedicine environment, cost of telecommunications, and persistent concerns about data privacy, patient safety, charging of professional and therapy fees, cost-effectiveness, and various medicolegal risks. ${ }^{17,18}$

Several studies had investigated the acceptance and use of speech telerehabilitation for patients with PD. ${ }^{19}$ One study done in Brazil investigated the efficacy of vocal telerehabilitation among 20 patients with PD aged 42-78 years. The results showed improvement in vocal pattern among the participants after the extended version of LSVT was used. The participants underwent teletherapy sessions twice a week for 8 weeks for a total of 16 sessions and reported satisfaction with and preference for telerehabilitation compared to traditional face-to-face rehabilitation because of the interactive dynamics, comfort, convenience, and independence. ${ }^{19}$ Similar results were seen in another study, wherein participants were highly satisfied with video conference-based telerehabilitation. Significant improvements were obtained in terms of sound pressure level for sustained vowels, monologue, speech intelligibility, and voice loudness after 12 sessions within 4 weeks of telerehabilitation. ${ }^{24}$

Praat $^{\mathrm{TM}}$ is a freely downloadable and open-source computer application that analyzes, synthesizes, and manipulates speech in phonetics. ${ }^{25}$ Through certain parameters, such as pitch defined as the range of tone (i.e., highness or lowness), shimmer (i.e., variation of amplitude or volume of the sound wave), jitter (i.e., variation of frequency or pitch of sound wave), harmonics to noise ratio (HNR), and noise to harmonics ratio (NHR), the application allows the clinician to determine abnormalities in phonation, such as hoarseness, asthenia, and limited pitch variation. The parameters are useful in providing objective quantitative data, as compared to subjective data obtained from mere perceptual assessment. The application also enables the clinician to record a sound with an audio input device and have a look "inside" the sound. ${ }^{26}$ More importantly, it allows for assessment of abnormalities in the speech of the patient, treatment planning, and outcomes monitoring. ${ }^{27}$ Praat ${ }^{\mathrm{TM}}$ had already been demonstrated in earlier studies to be a valid tool in the objective assessment of speech and voice characteristics. ${ }^{26,28,29}$

In conjunction with the use of Praat ${ }^{\mathrm{TM}}$, incorporating the use of vocal function exercises (VFE), pitch extension exercises, and choral singing therapy was also implemented. VFE refers to a set of systematic exercises used to improve vocal quality and phonation efficacy by addressing the strength and coordination of laryngeal muscles. ${ }^{30}$ Pitch extension exercises involve phonation drills at varied frequencies to strengthen the vocal folds and improve suprasegmental patterns (prosody), ${ }^{31,32}$ enabling the patient to better express herself in speaking engagements at home and online work-related activities. Meanwhile, choral singing therapy is based on the principle of overlapping areas of bi-hemispheric cortical activation to treat motor speech abnormalities associated with neurological disorders like PD. ${ }^{33}$

The patient's cognitive and functional skills, awareness of her voice quality, compliance with medical advice, and family support may be considered positive prognosticating factors for the success of the telerehabilitation program. There was no report of problems with the internet and audiovisual clarity in this case, given the usual bandwidth commonly available from home wi-fi, which usually averages 18.6 megabits per second (Mbps) in the Philippines. ${ }^{34}$ The patient's lapses with the prescribed home exercise program when she became busy at work, however, resulted in regression of achieved skills, emphasizing the need for continued synchronous teletherapy and compliance with asynchronous home exercises to ensure carry-over of therapy gains. To our knowledge, there is a research gap on the duration, intensity, and frequency of speech teletherapy to sustain long-term outcomes. Nonetheless, it seems that the success of any telerehabilitation program, as with a traditional face-to-face counterpart, relies heavily on patient compliance and family support.

\section{CONCLUSION}

Speech telerehabilitation is feasible for a patient with $\mathrm{PD}$ in a low-resource country to overcome the barriers to in-person rehabilitation, such as physical distancing due to COVID-19. Tele-evaluation of speech may be improved by incorporating objective measurement tools, such as the freely downloadable and easy-to-use Praat ${ }^{\mathrm{TM}}$ computer application, to aid in treatment planning and outcomes 
monitoring that can be appreciated by both patient and clinician. The improvements achieved through synchronous and asynchronous teletherapy can be better maintained by ensuring compliance to home exercise programs.

\section{Ethical Considerations}

The authors have registered the paper with their institutional review board and followed the principles outlined in the Declaration of Helsinki for human studies. In addition, recorded or electronic informed consent was obtained from the patient.

\section{Statement of Authorship}

All authors participated in the data collection and analysis and approved the final version submitted.

\section{Author Disclosure}

This study did not receive any specific grant or support from funding agencies in the public, commercial, or notfor-profit sectors. The authors had no conflicts of interest to disclose.

\section{Funding Source}

This study did not receive any specific grant or support from funding agencies in the public, commercial, or not-forprofit sectors. No external assistance for writing was necessary.

\section{REFERENCES}

1. Fayyaz M, Jaffery S, Anwar F, Zil-E-Ali A, Anjum I. The effect of physical activity in Parkinson's disease: a mini-review. Cureus. 2018 July; 10(7):e2995.

2. Bhalsing K, Abbas M, Tan L. Role of physical activity in Parkinson's disease. Ann Indian Acad Neurol. 2018 Oct; 21(4):242-9.

3. Brabenec L, Mekyska J, Galaz Z, Rektorova I. Speech disorders in Parkinson's disease: early diagnostics and effects of medication and brain stimulation. J Neural Transm. 2017 Jan; 124(3):303-34.

4. Ray Dorsey E, Elbaz A, Nichols E, Abd-Allah F, Abdelalim A, Adsuar $\mathrm{J}$, et al. Global, regional, and national burden of Parkinson's disease, 1990-2016: a systematic analysis for the Global Burden of Disease Study 2016. Lancet Neurol. 2018 Oct; 17(11):939-53.

5. Scott S, Caird FI. Speech therapy for Parkinson's disease. J Neurol Neurosurg Psychiatry. 1983; 46:140-4.

6. Ramig LO, Fox C, Sapir S. Speech treatment for Parkinson's disease. Expert Rev. Neurother. 2008; 8:297-309.

7. Herd CP, Tomlinson CL, Deane KH, Brady MC, Smith $\mathrm{CH}$, Sackley CM, et al. Speech and language therapy versus placebo or no intervention for speech problems in Parkinson's disease. Cochrane Database Syst Rev. 2012 Aug(8):CD002812.

8. Herd CP, Tomlinson CL, Deane KH, Brady MC, Smith CH, Sackley $\mathrm{CM}$, et al. Comparison of speech and language therapy techniques for speech problems in Parkinson's disease. Cochrane Database Syst Rev. 2012 Aug 15; 2012(8):CD002814.

9. Boersma P, Weenink D. Praat: doing phonetics by computer (version 5.0.20) [computer software] [Internet]. 2011 [cited 2021 Jul] Available from: www.praat.org.

10. Bowen LK, Hands GL, Pradhan S, Stepp CE. Effects of Parkinson's disease on fundamental frequency variability in running speech. J Med Speech Lang Pathol. 2013; 21:235-44.

11. Skodda S, Grönheit W, Mancinelli N, Schlegel U. Progression of voice and speech impairment in the course of Parkinson's disease: a longitudinal study. Parkinsons Dis. 2013:1-8.
12. Gündoğdu A, Akidil AÖ, Kotan D. Resolving speech disorders in Parkinson disease: our clinical experience with voice therapy. Biomed Res. 2017; 28:3313-7.

13. Ramig L, Sapir S, Fox C, Countryman S. Changes in vocal loudness following intensive voice treatment $\left(\mathrm{LSVT}^{\circledR}{ }^{\circledR}\right.$ ) in individuals with Parkinson's disease: a comparison with untreated patients and normal age-matched controls. Mov Disord. 2001; 16(1):79-83.

14. Mahler LA, Ramig LO, Fox C. Evidence-based treatment of voice and speech disorders in Parkinson disease. Curr Opin Otolaryngol Head Neck Surg. 2015; 23:209-15.

15. Jamora R, Miyasaki J. Treatment gaps in Parkinson's disease care in the Philippines. Neurodegener Dis Manag. 2017 Aug; 7(4):245-51.

16. LSVT Global. Locate an LSVT certified clinician [Internet]. 2020 [cited 2020 Sep] Available from: https://www.lsvtglobal.com/ LSVTFindClinicians.

17. Leochico C, Espiritu A, Ignacio S, Mojica J. Challenges to the emergence of telerehabilitation in a developing country: a systematic review. Front Neurol. 2020 Sept; 11:1007.

18. Leochico C. Adoption of telerehabilitation in a developing country before and during the COVID-19 pandemic. Ann Phys Rehabil Med. 2020 June; 63(6):563-4.

19. Dias AE, Limongi JCP, Barbosa ER, Hsing WT. Voice telerehabilitation in Parkinson's disease. CoDAS. 2016; 28:176-81.

20. Styler W. Using Praat for linguistic research [Internet]. 2017 [cited 2021 Jul]. Available from: https://wstyler.ucsd.edu/praat/ UsingPraatforLinguisticResearchLatest.pdf.

21. Belsky MA, Shelly S, Rothenberger SD, et al. Phonation resistance training exercises (PhoRTE) with and without expiratory muscle strength training (EMST) for patients with presbyphonia: a noninferiority randomized clinical trial.J Voice. 2021; published ahead of print. doi:10.1016/j.jvoice.2021.02.015.

22. Eckel FC, Boone DR. The $\mathrm{S} / \mathrm{Z}$ ratio as an indicator of laryngeal pathology. J Speech Hear Disord. 1981; 46:147-9.

23. Leochico CFD, Mojica JAP, Rey-Matias RR, Supnet IE, Ignacio SD. Role of telerehabilitation in the Rehabilitation Medicine training program of a COVID-19 referral center in a developing country. Am J Phys Med Rehabil. 2021 Jun; 100(6):526-32.

24. Chan M, Chu S, Ahmad K, Ibrahim N. Voice therapy for Parkinson's disease via smartphone videoconference in Malaysia: a preliminary study. J Telemed Telecare. 2019 July; 0(0):1-9.

25. Maryn Y. Practical acoustics in clinical voice assessment: a Praat primer. Perspect. ASHA Spec Interest Groups. 2017; 2(3):14-32.

26. Alam N, Sinha V, Kumar SS, Katarkar A, Jain A. Efficacy of voice therapy for treatment of puberphonia: review of 20 cases. World Articles in Ear, Nose and Throat. 2012; 5(1).

27. Boersma B, Heuven V. Speak and unspeak with PRAAT. Glot International. 2001 Dec; 5(9):341-7.

28. Mezzedimi C, Di Francesco M, Livi W, Spinosi M, De Felice C. Objective evaluation of presbyphonia: spectroacoustic study on 142 patients with Praat. J Voice. 2017 Mar; 31(2), 257: e25-257, e32.

29. Latoszek B. Treatment effectiveness of Novafon local vibration voice therapy for dysphonia treatment. J Voice. 2020 Jan; 34(1), 160: e7-160, e14.

30. American Speech-Language-Hearing Association. Voice disorders: practice portal [Internet]. (n.d.) [cited 2020 Nov] Available from: www.asha.org/Practice-Portal/Clinical-Topics/Voice-Disorders/.

31. Veenendaal NJ, Groen MA, Verhoeven L. The contribution of segmental and suprasegmental phonology to reading comprehension. Read Res Q. 2016; 51(1):55-66.

32. Chan A, Mccabe P, Madill C. The implementation of evidence-based practice in the management of adults with functional voice disorders: a national survey of speech-language pathologists. Int J SpeechLang. 2013 Jun; 15(3):334-44.

33. Higgins A, Richardson K. The effects of a choral singing intervention on speech characteristics in individuals with Parkinson's disease: an exploratory study. Commun Disord. 2018 Jul; 40(4):195-205.

34. Eastvantage. Philippines's internet speed rank 2020 [Internet]. 2020 [cited 2020 Nov] Available from: https://www.eastvantage.com/ newsroom/philippines-internet-speed-rank-2020. 